\title{
HA/HSA co-modified erlotinib-albumin nanoparticles for lung cancer treatment
}

This article was published in the following Dove Press journal:

Drug Design, Development and Therapy

\section{Yuzhou Shen \\ Wentao Li}

Department of Thoracic Surgery, Shanghai Chest Hospital, Shanghai Jiao Tong University, Shanghai 200030, China
Correspondence: Wentao Li

Department of Thoracic Surgery,

Shanghai Chest Hospital, Shanghai Jiao

Tong University, 24I Huaihai West Road,

Shanghai 200030, China

Email wentaolidr@2Icn.com
Tel/fax +86 2l 22200000

Background: Aim of this study was to prepare the hyaluronic acid and human serum albumin modified erlotinib nanoparticles (ERT-HSA-HA NPs) delivery system by a precipitation method.

Methods: ERT-HSA-HA NPs were characterized for physical properties, such as morphology and particle size, and in vitro drug release. Moreover, the cytotoxicity, cellular uptake, in vivo studies of ERT-HSA-HA nanoparticle were investigated and compared in A549 cells.

Results: The ERT-HSA-HA NPs showed spherical morphology, and their hydrodynamic diameter was $112.5 \pm 2.8 \mathrm{~nm}$. The drug loading amount and encapsulation efficiency were $5.6 \%$ and $81.2 \%$, respectively. After 3 months of storage, no dramatic change, such as visible aggregation, drug content changes, and precipitation, in the appearance of ERT-HSA-HA NPs occurred. In vitro release showed that the release of ERT from HSA-HA NPs was slow, without obvious burst effects at an early stage. In in vivo studies, ERT-HSA-HA NPs showed a superior antiproliferative effect on A549 cells, and the HA modification strategy can also facilitate the high-efficiency uptake of ERT-HSA NPs by A549 cells. Pharmacokinetic studies showed that the form of NPs could significantly extend the role of ERT in vivo (provided higher bioavailability). However, there was no significant difference in the pharmacokinetic parameters between ERT-HSA NPs and ERT-HSA-HA NPs after intravenous administration. In terms of in vivo antitumor activity, ERT-HSA-HA NP-treated mice showed a significantly suppressed tumor growth and no relapse after $30 \mathrm{~d}$ of treatment.

Conclusion: HA/HSA co-modified erlotinib albumin nanoparticles was expected to be a new strategy in the treatment of lung cancer.

Keywords: erlotinib, hyaluronic acid, human serum albumin, nanoparticles, pharmacokinetic, antitumor activity

\section{Introduction}

Erlotinib (ERT) is an effective selective tyrosine kinase inhibitor. It reversibly binds the binding site of ATP in the epidermal growth factor receptor tyrosine kinase domain to inhibit the automatic phosphorylation of tyrosine kinases. ${ }^{1,2}$ Inhibition of tyrosine kinase promotes cell apoptosis, inhibits angiogenesis, and ultimately prevents proliferation of cells. ${ }^{3-5}$ ERT, which is used in the treatment of various solid tumors such as non small-cell lung cancer, is available in oral form. ${ }^{6,7}$ The new ERT delivery system is limited due to its poor solubility. Thus, great effort is currently devoted to the design of novel systems for targeted ERT delivery. The development of targeting moieties to selectively internalize the drug payload inside the cancer cells is now considered an important step. ${ }^{8}$ For example, it can improve poor solubility and low bioavailability, reduce rapid renal clearance, and improve cell selectivity. ${ }^{9}$ At present, many encapsulation methods that use delivery systems such as liposomes, polymeric-based nanoparticles (NPs), hydrogels, and serum albumin are used. ${ }^{10-13}$ Among the wide range

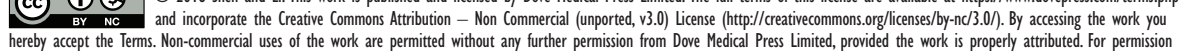

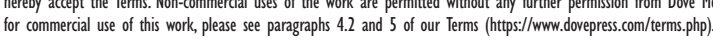


of targeting ligands, hyaluronic acid (HA) and human serum albumin (HSA) have proven success in lung cancer targeting within tumor cell lines and animal models. ${ }^{14,15}$

HSA, an endogenous protein, is nontoxic, is nonimmunogenic, and has good biocompatibility. ${ }^{16}$ It has been widely used in drug delivery as a macromolecular protein carrier. ${ }^{17}$ Therefore, HSA can improve the solubility of lipophilic drugs. In addition, the presence of carboxylic and amino groups on the surface promotes the surface functionalization for albumin NPs. ${ }^{18,19}$ For example, the surface of albumin NPs can be modified with fluorescent dyes, target molecules, and functional RNA by covalent binding. ${ }^{20,21}$ It also makes it easy to use hydrophilic polymers, such as polyethylene glycol, to prolong circulation. $^{22}$

HA, a linear negatively charged polysaccharide consisting of 2 alternating units of D-glucuronic acid and $N$-acetyl-Dglucosamine, is being investigated as a targeting constituent of drug carriers for cancer therapy. HA is known to interact with CD44 (cluster of differentiation-44), receptor for hyaluronanmediated motility, and lymphatic vessel endothelial receptor-1 cell surface receptors, which are overexpressed in tumor cells. ${ }^{23-25}$ Activation of such receptors, upon HA-receptor interaction, can stimulate signal responses that promote internalization of chemotherapeutic agent-loaded NPs. ${ }^{26}$

This study aimed to prepare the ERT-HSA-HA NP delivery system by a precipitation method. ERT-HSA-HA NPs were characterized in terms of physical properties, such as morphology and particle size, and in vitro drug release. Moreover, the cytotoxicity, cellular uptake, and in vivo activity of ERTHSA-HA NPs were investigated and compared in A549 cells.

\section{Materials and methods Materials}

ERT was a gift from Fuan Pharma Co Ltd (Chongqing, China). HSA ( $>99 \%$ purity) was purchased from Sigma-Aldrich ( $\mathrm{St}$ Louis, MO, USA). HA (molecular weight [Mw]: 5,400 Da) was kindly provided by Bloomage Freda Biochem Co Ltd (Jinan, China). A549 cells were purchased from Cobioer Biotechnology Co Ltd (Nanjing, China). All other reagents were obtained from Sinopharm Chemical Reagent Co Ltd (Shanghai, China). Methanol and acetonitrile (chromatographic grade) were obtained from Sigma-Aldrich. Deionized water used throughout the research work was produced in the laboratory using a Milli-Q Gradient A10 System.

\section{Preparation of ERT-HSA-HA NPs}

To prepare ERT-HSA NPs, HSA (20 mg) and ERT ( $5 \mathrm{mg}$ ) were dissolved in $10 \mathrm{~mL}$ dichloromethane. The solvent was then evaporated under reduced pressure at $40^{\circ} \mathrm{C}$, yielding a thin and transparent film containing HSA and ERT. The film was rehydrated in deionized water and sonicated to disperse into selfassembled NPs. The final solution was filtered through a $0.8-\mu \mathrm{m}$ cellulose acetate filter unit, freeze-dried, and stored at $-20^{\circ} \mathrm{C}$ until needed. The freshly prepared ERT-HSA NPs were surface decorated by HA via electrostatic deposition technique. ${ }^{27,28}$ For the surface-coating process, $5 \mathrm{~mL}$ of ERT-HSA NP dispersion $(0.05 \%, \mathrm{w} / \mathrm{v})$ was added into an equal volume of HA solution under magnetic stirring at $750 \mathrm{rpm}$ for $30 \mathrm{~min}$.

\section{Characterization of the prepared ERT-HSA-HA NPs}

For particle size analysis, a dynamic light scattering technique was applied, whereas laser Doppler anemometry was used for zeta potential measurements using a Zetasizer Nano ZS instrument (Malvern Instruments Ltd, Malvern, UK). All measurements were performed in triplicate, and the values are represented as mean $\pm \mathrm{SD}(\mathrm{n}=3)$. The morphologies of the NPs were visualized by transmission electronic microscopy (JEM1200EX; JEOL, Tokyo, Japan). The samples were added to the surface of copper grids and stained with phosphotungstic acid $(1 \%, \mathrm{w} / \mathrm{v})$. The accelerating voltage was $120 \mathrm{kV}$.

Drug loading (\% DL)

$$
=\frac{\text { Amount of drug entrapped }}{\text { Total weight of NPs used in formulation }} \times 100 \text {. }
$$

The entrapment efficiency $(\% \mathrm{EE})$ was determined by measuring the concentration of unentrapped drug in the NP dispersion. Briefly, the NP dispersion was subjected to centrifugation for $20 \mathrm{~min}$ at $4^{\circ} \mathrm{C}$ at $4,500 \mathrm{rpm}$, and the amount of ERT in the supernatant was determined at $332 \mathrm{~nm}$ by high-performance liquid chromatography (HPLC) after dissolving the supernatant in acetone and ethanol (1:1) mixture. The drug encapsulation efficiency was calculated using the following equation:

$$
\% \mathrm{EE}=\frac{\text { ERT loaded in the NPs }}{\text { Weight of the total NPs }}
$$

\section{Stability study}

According to International Conference on Harmonization guidelines, the particle size, zeta potential, EE, and DL of ERT-HSA-HA NPs were evaluated at $4^{\circ} \mathrm{C}$ for 3 months as the chemical and physical stability. The centrifuge tests were also carried out to assess the physical stability of the NP solutions. The NP solutions were centrifuged for $30 \mathrm{~min}$ at $650 \times g$ in the centrifuge tests. 


\section{In vitro release}

In vitro studies of drug release from modified and unmodified ERT-loaded HSA NPs were carried out for $24 \mathrm{~h}$ in PBS (pH 7.4). An aliquot of NPs, containing a fixed amount of ERT $(10 \mathrm{mg}$ ), was introduced in a dialysis bag (Mw cutoff $10,000-12,000 \mathrm{Da}$ ) and then placed in PBS adjusted at $37^{\circ} \mathrm{C}$ under gentle magnetic stirring $(75 \mathrm{rpm})$ in a volume $(20 \mathrm{~mL}$, $0.5 \%$ Tween-80) achieving the sink condition. At scheduled time intervals, $1 \mathrm{~mL}$ of medium was withdrawn and replaced with an equal volume of fresh medium. The amount of ERT released was calculated by HPLC at $332 \mathrm{~nm}$. The whole experimental procedure was performed in triplicate. The release of the free drug was also studied in the same way.

The ERT release profiles of modified and unmodified NPs were compared using the similarity factor $\left(f_{2}\right)$ according to the equation reported, ${ }^{29}$ $f_{2}=50 \log \left\{\left[1+\frac{1}{n} \sum_{t=1}^{n}(R t-T t)^{2}\right]^{-0.5} \times 100\right\}$ where "log" is the logarithm to base 10 , " $n$ " is the number of sampling time points, " $P$ " is the summation over all time points, and " $R t$ " and " $T t$ " are the cumulative percentages of drug released at each of the " $n$ " time points of the reference and test products, respectively. Further, $f_{2}$ values between 50 and 100 guarantee likeness of the 2 dissolution profiles and, hence, the performance of the 2 formulae.

\section{In vitro anticancer activity assay}

The in vitro anticancer activity of ERT-HSA-HA NPs on human lung carcinoma A549 cells was evaluated by the MTT assay. Control experiments were carried out using a complete growth culture medium, only as a nontoxic control. A549 cells $\left(1 \times 10^{5}\right.$ cells per well in $1 \mathrm{~mL}$ medium) were seeded into 96-well plates. After $24 \mathrm{~h}$, cells were incubated with different samples containing varying concentrations of ERT-HSA-HA NPs, ERT-HSA NPs, or free ERT or blank NPs (from $0.002 \mu \mathrm{g} / \mathrm{mL}$ to $20 \mu \mathrm{g} / \mathrm{mL}$ ). After $48 \mathrm{~h}$ of incubation under cell culture conditions, the growth and viability of cells were determined by using MTT assay. Then, $1 \mathrm{~mL}$ complete growth culture medium and $60 \mu \mathrm{L}$ of MTT solution ( $5 \mathrm{mg} / \mathrm{mL}$ in PBS) were added to each well and incubated for $4 \mathrm{~h}$. The absorbance was determined using a microplate reader at $540 \mathrm{~nm}$. The results were expressed as percentages relative to the result obtained with a nontoxic control.

\section{Cellular uptake}

The cellular internalization of free ERT, ERT-HSA NPs, and ERT-HSA-HA NPs was visualized by confocal microscopy using Coumarin-6 as a fluorescent probe. A549 cells were grown in Roswell Park Memorial Institute 1640 medium supplemented with $10 \%(\mathrm{v} / \mathrm{v}) \mathrm{FBS}$ and $5 \%$ antibiotics (100 IU/ $\mathrm{mL}$ of penicillin $\mathrm{G}$ sodium and $100 \mu \mathrm{g} / \mathrm{mL}$ of streptomycin sulfate). A549 cells were inoculated in a cell culture dish at of $4 \times 10^{5}$ cells per dish for initial density. Cells were then incubated with Coumarin-6-adsorbed free ERT, ERT-HSA NPs, and ERT-HSA-HA NPs (equivalent to $0.1 \mu \mathrm{g} / \mathrm{mL}$ of Coumarin-6) for $2 \mathrm{~h}$ at $37^{\circ} \mathrm{C} \pm 0.5^{\circ} \mathrm{C}$.

Subsequently, cells were washed several times with PBS and fixed with $4 \%$ paraformaldehyde for $10 \mathrm{~min}$. Finally, cells were observed under a confocal microscope. For quantitative estimates of ERT uptake, the density of cells inoculated on $24-w e l l$ plates was $3 \times 10^{4}$ cells. When they reached $70 \%-80 \%$ confluence, cells were incubated with Coumarin-6-adsorbed free ERT, ERT-HSA NPs, and ERTHSA-HA NPs (equivalent to $0.1 \mu \mathrm{g} / \mathrm{mL}$ of Coumarin-6). After $2 \mathrm{~h}$ of culture, cells were washed several times with cold PBS. Subsequently, cells were dissolved by addition of Triton X-100 (0.1\%). Fluorescence intensities were measured by a multimode microplate reader at an excitation wavelength of $440 \mathrm{~nm}$ and an emission wavelength of $520 \mathrm{~nm}$.

\section{Pharmacokinetic studies}

All the in vivo experimental protocols were approved by the animal care committee of Shanghai Jiao Tong University, and all experiments were conducted in strict accordance with the Laboratory Animal Care and Use guidelines, adopted by the National Institutes of Health (Shanghai, China). SpragueDawley rats weighing 200-220 g were used for the pharmacokinetic study. Before the experiment was commenced, the rats were kept in a state of fasting for $6 \mathrm{~h}$. In the experiment, all rats were divided into 3 groups ( 8 rats per group). One group was given ERT injection (free drug, as a control) and the other 2 groups were given modified and unmodified ERT-loaded HSA NPs (dose $=10 \mathrm{mg} / \mathrm{kg}$ ). All the formulations were introduced into rats via intravenous injection. Blood samples $(2 \mathrm{~mL})$ were collected from the tail vein into heparinized $5 \mathrm{~mL}$ polythene tubes before administration and at $0.25,0.5,1,2,4,6,8,10,12$, and $24 \mathrm{~h}$ after dosing. The collected plasma was stored at $-20^{\circ} \mathrm{C}$ until analysis.

\section{In vivo antitumor activity}

The A549 model was established as described before. ${ }^{15}$ The treatments were started on the day when the tumor volume reached $100-150 \mathrm{~mm}^{3}$, which was set as Day 0 . On Day 0 , the mice were randomly divided into 4 groups (blank NPs, free ERT, ERT-HSA NPs, and ERT-HSA-HA NPs) $(n=10)$. Samples, at a dose of $10 \mathrm{mg} / \mathrm{kg}$, were injected intravenously via the tail vein every $3 \mathrm{~d}, 4$ administrations in total. During 
the study, the tumor size and body weight were measured 3 times in $1 \mathrm{wk}$. On Day 30, the mice were sacrificed and resected to weigh the tumor. The test drug administration procedures were as follows: Day 0, Day 3, Day 9, Day 12, and euthanasia on Day 30. A digital caliper was used to measure the tumor diameters (in millimeters), and tumor volumes (in cubic millimeters) were calculated using the following formula: tumor volume=length $\times$ widt ${ }^{2} \times 0.5$. In the course of the study, mice were regularly weighed to monitor the potential toxicities.

\section{Statistical analysis}

All data points were denoted as mean \pm SD. In order to determine statistical significance, analysis of variance (1-way or 2-way) was conducted using GraphPad Prism 6 . The difference was considered statistically significant if $p \leq 0.05$.

\section{Results and discussion Characterization of NPs}

ERT is a novel small-molecule inhibitor for the intracellular ATP-binding site of EGFR tyrosine kinase. Currently, it is clinically applied as the first-line treatment for lung cancer. In our previous study, we successfully developed self-assembled ERT NPs in an aqueous medium ( 120 nm, Unpublished data 2016) with an HSA-HA conjugate using a solvent-free film casting and rehydration method. Hydrophobic ERT is expected to be localized at the hydrophobic domains of HSA-HA and may be exposed during the solvent evaporation process at an elevated temperature. The entrapment might effectively prevent the formation and growth of drug crystals in the membrane. The surface of the NPs would be stabilized by the HA segments that form the outermost hydrophilic layer. The ERT-HSA-HA NPs were spherical in shape, with a hydrodynamic diameter of $112.5 \pm 2.8 \mathrm{~nm}$ (Figure 1A). The DL amount and EE were 5.6\% and 81.2\%, respectively. Table 1 also gives the stability data of particle size of ERT-HSA-HA NPs stored at $4^{\circ} \mathrm{C}$. After 3 mo of storage, no dramatic change (such as visible aggregation, drug content changes, and precipitation) in the appearance of ERT-HSA-HA NPs occurred. TEM images (Figure 1B) showed that NPs were dispersed well and had uniform shape. No significant change in clarity and phase separation was observed in the tests of centrifugation.

\section{In vitro release}

The cumulative amounts of ERT released from the HAmodified ERT-HSA NPs are illustrated in Figure 2. A control experiment with free ERT was also adopted under comparable conditions, in which total drug diffusion was achieved across the dialysis membrane within 2 h. For HA-modified ERTHSA NPs, a low ERT burst release of 5\% was noted at $1 \mathrm{~h}$. An initial burst of drug release was obvious for unmodified NPs, attributed to the rapid diffusion of the free drug adsorbed on the particles. The slower release rate of ERT from HSA-HA NPs in comparison to unmodified ones, with an extension in drug release rate over $8 \mathrm{~h}$, after which a plateau was observed until $12 \mathrm{~h}$, may be ascribed to the surface-adsorbed coating layer, which might hinder the diffusion of the entrapped drug in the nanosystem. Applying the similarity factor $\left(f_{2}\right)$, we found a low similarity in the release patterns of ERT from HSA-HA NPs and HSA NPs with $f_{2}=62$. It might be explained by the enhanced penetrability of short-chain HA within the pores of NPs, which would result in more compact
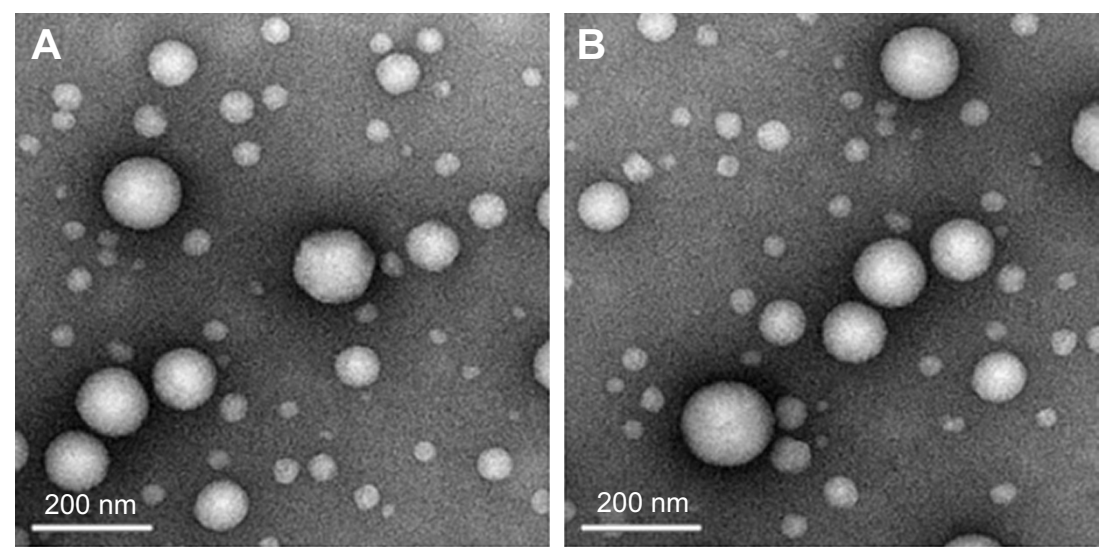

Figure I Transmission electron microscopy of ERT-HSA-HA NPs.

Notes: (A) Day 0, (B) 3 months later (Magnification: $\times 100,000$.)

Abbreviation: ERT-HSA-HA NPs, hyaluronic acid- and human serum albumin-modified erlotinib nanoparticles. 
Table I The stability data of ERT-HSA-HA NPs before and after storage at $4^{\circ} \mathrm{C}$

\begin{tabular}{lllllll}
\hline Nanoparticles & Time & $\begin{array}{l}\text { Particle } \\
\text { size }(\mathbf{n m})\end{array}$ & $\begin{array}{l}\text { Zeta potential } \\
(\mathbf{m V})\end{array}$ & $\begin{array}{l}\text { Encapsulation } \\
\text { efficiency (\%) }\end{array}$ & $\begin{array}{l}\text { Drug } \\
\text { loading (\%) }\end{array}$ & $\begin{array}{l}\text { Polydispersity } \\
\text { index }\end{array}$ \\
\hline ERT-HSA-HA NPs & Od & $112.5 \pm 2.8$ & $-21.2 \pm 3.2$ & $81.2 \pm 2.7$ & $5.6 \pm 0.4$ & $<0.25$ \\
& I mo & $114.2 \pm 3.5$ & $-22.5 \pm 3.3$ & $82.4 \pm 1.9$ & $5.3 \pm 0.3$ & $<0.24$ \\
& $2 \mathrm{mo}$ & $115.1 \pm 3.2$ & $-24.1 \pm 2.7$ & $80.1 \pm 2.2$ & $5.4 \pm 0.2$ & $<0.28$ \\
& $3 \mathrm{mo}$ & $114.9 \pm 3.9$ & $-23.4 \pm 2.6$ & $80.3 \pm 1.2$ & $5.2 \pm 0.5$ & $<0.26$ \\
\hline
\end{tabular}

Note: $n=3$.

Abbreviation: ERT-HSA-HA NPs, hyaluronic acid- and human serum albumin-modified erlotinib nanoparticles.

particles with slower drug release rates owing to the crosslinking efficiency of the polyanionic HA.

\section{In vitro anticancer activity assay}

After the formulation of ERT-HSA-HA NPs had been optimized, we assessed the anticancer efficacy of ERT-HSA-HA NPs on A549 cells. The cells were exposed for $96 \mathrm{~h}$ to different concentrations of ERT-HSA-HA NPs, ERT-HSA NPs, or free ERT or blank NPs. The survival profile is presented in Figure 3. The negative control was represented by blank NPs, for which cell survival is $100 \%$. This result showed the safety of the unloaded vector. ERT-HSA NPs and free ERT have similar cytotoxicity characteristics, as expected; as the ERT concentration increased, greater cell death was observed. Surprisingly, ERT-HSA-HA NPs showed a superior antiproliferative effect on A549 cells, which may inhibit multidrug resistance, as well as having an obvious synergistic effect.

\section{Cellular uptake}

ERT-HSA NPs and ERT-HSA-HA NPs were labeled with a fluorescent probe. As shown in Figure 4, the nuclei, which

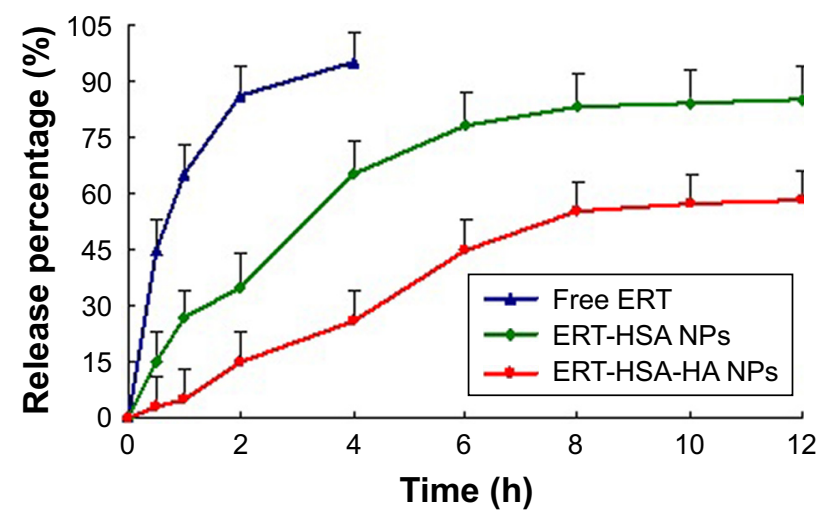

Figure 2 The release profiles of free ERT, ERT-HSA NPs, and ERT-HSA-HA NPs $(n=6)$.

Abbreviations: ERT, erlotinib; ERT-HSA NPs, human serum albumin-modified erlotinib nanoparticles; ERT-HSA-HA NPs, hyaluronic acid- and human serum albumin-modified erlotinib nanoparticles. were stained by Coumarin-6, displayed green fluorescence. A strong green fluorescence was observed in the perinuclear region of A549 cells treated with ERT-HSA-HA NPs, indicating that a sufficient amount of ERT-HSA-HA NPs entered the cytoplasm. In contrast, the green fluorescence shown in ERT-HSA NP-treated A549 cells was rare. Moreover, A549 cells pretreated with free ERT also exhibited mild green fluorescence, likely because the ability to cross cells is weak. The cellular uptake ratio of the NPs was detected by flow cytometry, which was $8.6 \% \pm 1.4 \%, 16.3 \% \pm 2.4 \%$, and $62.3 \% \pm 4.7 \%$ for free ERT-, ERT-HSA NP-, and ERT-HSA-HA NP-treated A549 cells, respectively. These results demonstrate that the HA modification strategy can facilitate the high-efficiency uptake of ERT-HSA NPs by A549 cells.

\section{Pharmacokinetic studies}

Figure 5 shows the blood circulation time of free ERT, ERT-HSA NPs, and ERT-HSA-HA NPs after intravenous injection into mice. It can be seen that ERT-HSA NPs and

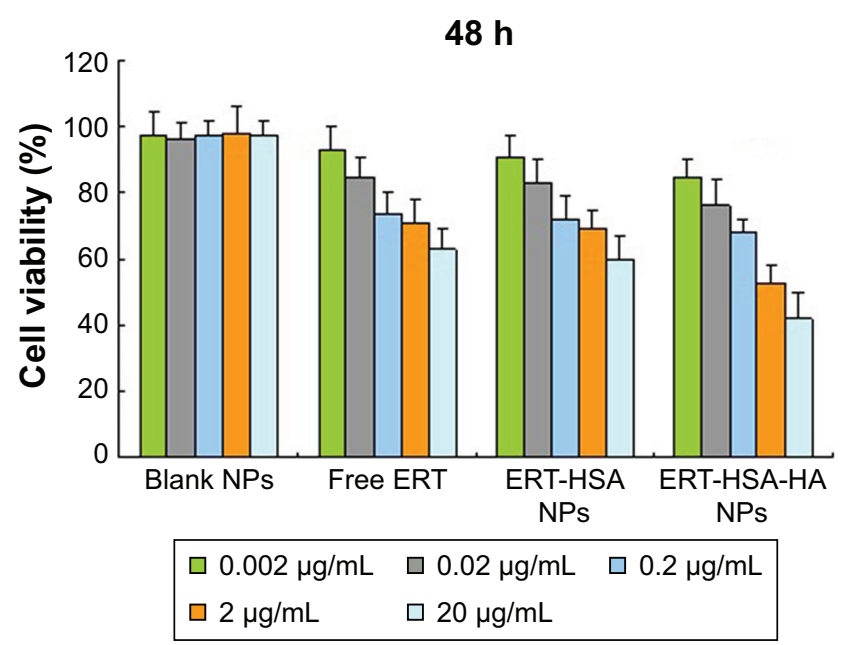

Figure 3 In vitro anticancer activity assay of ERT-HSA-HA NPs, ERT-HSA NPs, or free ERT or blank NPs on A549 cell lines.

Note: Cell viability was assessed by the MTT assay.

Abbreviations: ERT, erlotinib; ERT-HSA NPs, human serum albumin-modified erlotinib nanoparticles; ERT-HSA-HA NPs, hyaluronic acid- and human serum albumin-modified erlotinib nanoparticles. 

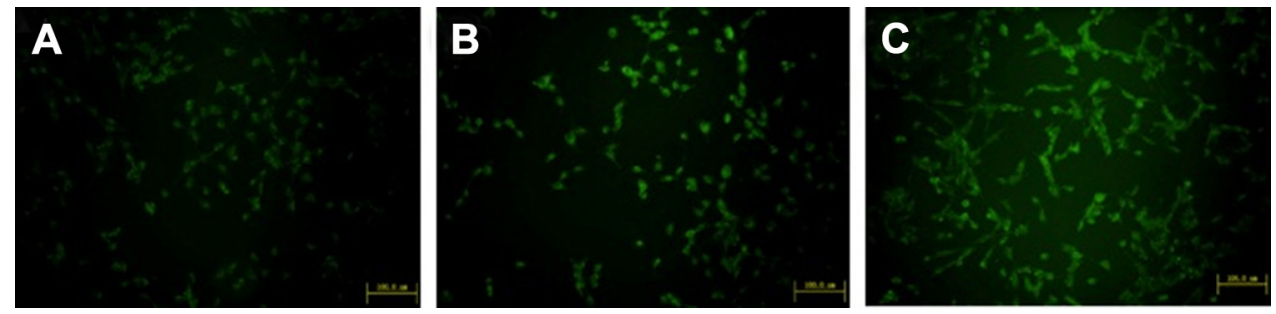

Figure 4 Confocal images of cellular uptake of (A) free ERT, (B) ERT-HSA NPs, and (C) ERT-HSA-HA NPs by A549 cells.

Note: Incubation time was $2 \mathrm{~h}$.

Abbreviations: ERT, erlotinib; ERT-HSA NPs, human serum albumin-modified erlotinib nanoparticles; ERT-HSA-HA NPs, hyaluronic acid- and human serum albuminmodified erlotinib nanoparticles.

ERT-HSA-HA NPs have nearly the same half-life $\left(t_{1 / 2}\right)$ of $91.2 \pm 7.9 \mathrm{~min}$ and $96.4 \pm 8.3 \mathrm{~min}$, respectively (Table 2 ), while free ERT was quickly removed from the blood circulating system with $t_{1 / 2}=38.3 \pm 7.2 \mathrm{~min}$. After the single injection, the plasma drug concentration quickly reached the maximum $(105.9 \pm 12.1 \mu \mathrm{g} / \mathrm{mL})$ in $15 \mathrm{~min}$ and then it decreased rapidly, leaving around $10 \%$ of the maximum serum concentration $\left(C_{\max }\right)$ value $2 \mathrm{~h}$ later, which implied that rapid in vivo elimination of ERT occurs in rats. In the case of intravenous administration, the in vivo profile of ERT NPs was smoother than the ERT-only injection group. ERT-HSA-HA NPs prolonged the blood circulation time of ERT, approximately increasing it by 2.52 folds $\left(t_{1 / 2}\right)$. This phenomenon is mainly because the smaller particle size can significantly reduce the affinity of macrophages, thus prolonging the half-life of ERT in vivo. The area under the curve $\left(\mathrm{AUC}_{0-\infty}\right)$ of ERT-HSA-HA NPs was 3.8-fold higher compared with that of the free drug. Thus, it was reasonable to conclude that the NPs could significantly extend the role of ERT in vivo (provided higher bioavailability). However,

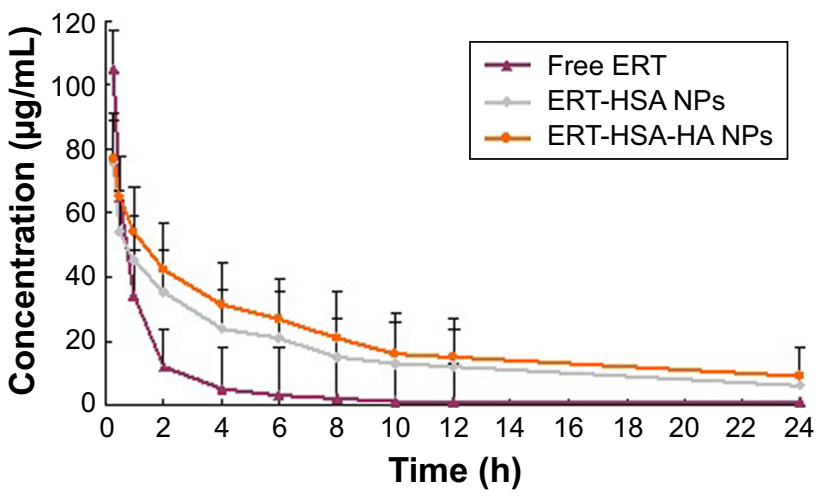

Figure 5 Blood circulation curves of free ERT, ERT-HSA NPs, and ERT-HSA-HA NPs in rats after intravenous injection.

Note: $\mathrm{n}=6$.

Abbreviations: ERT, erlotinib; ERT-HSA NPs, human serum albumin-modified erlotinib nanoparticles; ERT-HSA-HA NPs, hyaluronic acid- and human serum albumin-modified erlotinib nanoparticles. there was no significant difference in the pharmacokinetic parameters between ERT-HSA NPs and ERT-HSA-HA NPs after intravenous administration.

\section{In vivo antitumor activity}

Figure 6A shows the antitumor efficiency of free ERT, ERTHSA NPs, and ERT-HSA-HA NPs at the same concentration of ERT after intravenous injection via the tail. Mice were also treated with blank NPs. ERT-HSA-HA NP-treated mice showed a significantly inhibited tumor growth and no recurrence after $30 \mathrm{~d}$ of treatment, although the free ERT and ERT-HSA NP-treated groups, similar to the control group, showed ever-increasing tumor growth. As expected, the body weight of the mice in each group did not decrease significantly in $30 \mathrm{~d}$ of treatment (Figure 6B). In addition, after $30 \mathrm{~d}$ of treatment, H\&E staining of the main organs (heart, liver, spleen, lung, and kidney) was conducted for further assessment of systemic toxicity in vivo. No obvious tissue toxicity or abnormality was found in the tissue H\&Estained images of all the test groups, which further showed the safety of ERT-HSA-HA NPs in biomedical applications (data not shown).

Table 2 Pharmacokinetic parameters of ERT after intravenous administration of free ERT, ERT-HSA NPs, and ERT-HSA-HA NPs to rats

\begin{tabular}{llll}
\hline Parameters & \multicolumn{3}{l}{ Intravenous administration } \\
\cline { 2 - 4 } & Free ERT & \multicolumn{1}{l}{ ERT-HSA } & \multicolumn{1}{l}{ ERT-HSA- } \\
& & NPs & HA NPs \\
\hline$t_{1 / 2}(\mathrm{~min})$ & $38.3 \pm 7.2$ & $91.2 \pm 7.9 *$ & $96.4 \pm 8.3^{*}$ \\
$\mathrm{AUC}_{0-\mathrm{t}}(\mu \mathrm{g} \cdot \mathrm{min} / \mathrm{mL})$ & $129.1 \pm 12.3$ & $391.5 \pm 34.3^{*}$ & $496.2 \pm 45.3^{*}$ \\
$\mathrm{AUC}_{0-\infty}(\mu \mathrm{g} \cdot \mathrm{min} / \mathrm{mL})$ & $143.6 \pm 15.3$ & $432.3 \pm 47.2^{*}$ & $546.3 \pm 49.8^{*}$ \\
$\mathrm{MRT}(\mathrm{min})$ & $26.4 \pm 3.9$ & $69.3 \pm 7.2^{*}$ & $78.3 \pm 9.1^{*}$ \\
$\mathrm{CL}(\mathrm{L} / \mathrm{kg} / \mathrm{min})$ & $0.32 \pm 0.12$ & $0.09 \pm 0.02^{*}$ & $0.08 \pm 0.03^{*}$ \\
\hline
\end{tabular}

Notes: $* \mathrm{p}<0.05$ vs free ERT; $\mathrm{n}=6$.

Abbreviations: ERT, erlotinib; ERT-HSA NPs, human serum albumin-modified erlotinib nanoparticles; ERT-HSA-HA NPs, hyaluronic acid- and human serum albumin-modified erlotinib nanoparticles; $t_{1 / 2}$, half-life; AUC, area under the curve; MRT, mean residence time; $C L$, clearance. 

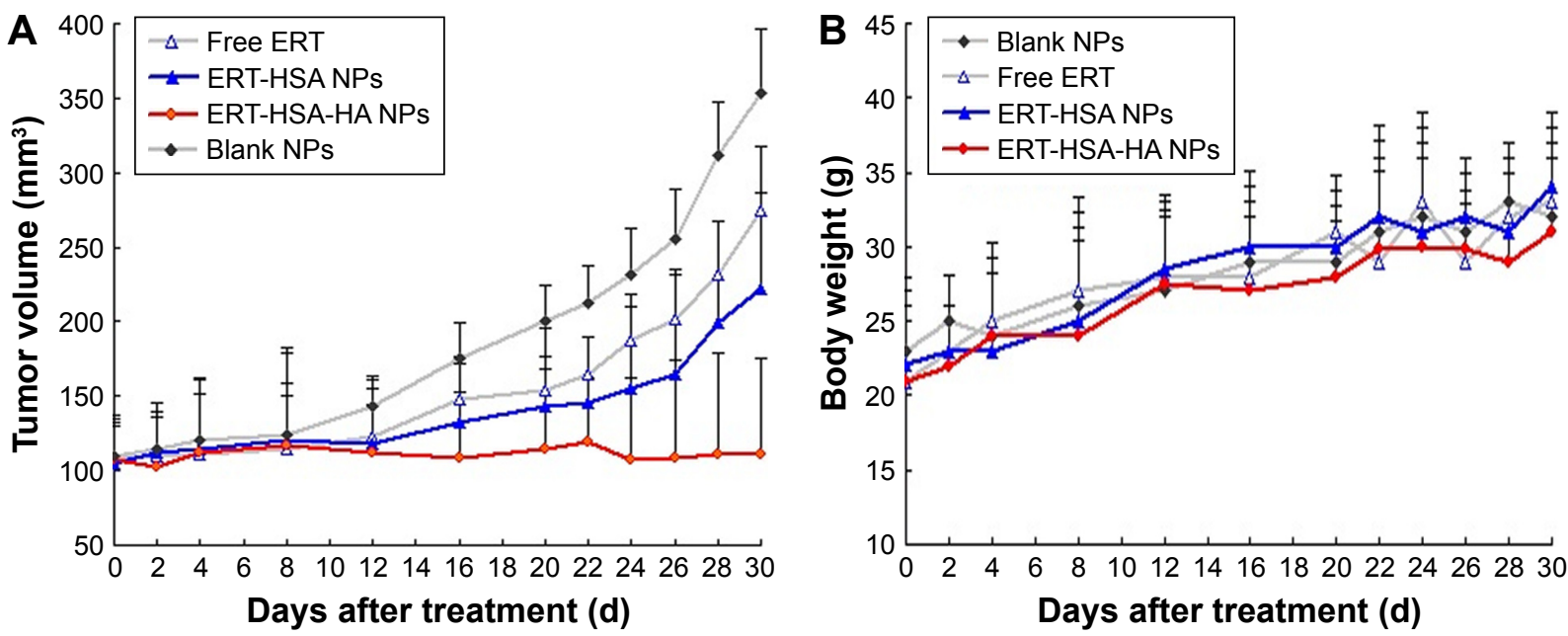

Figure 6 (A) The relative tumor volumes and (B) body weights of tumor-bearing mice after intravenous injection with blank NPs (control), free ERT, ERT-HSA NPs, and ERT-HSA-HA NPs.

Abbreviations: ERT, erlotinib; ERT-HSA NPs, human serum albumin-modified erlotinib nanoparticles; ERT-HSA-HA NPs, hyaluronic acid and human serum albuminmodified erlotinib nanoparticles.

\section{Conclusion}

HA/HSA co-modified erlotinib albumin nanoparticles was expected to be a new strategy in the treatment of lung cancer.

\section{Acknowledgments}

This study was supported by grants from the Medical Research Foundation of Jiangsu Provincial Health and Family Planning Commission (H201521) and the Natural Science Foundation of Jiangsu Provincial (BK20161224).

\section{Disclosure}

The authors report no conflicts of interest in this work.

\section{References}

1. Aydiner A, Yildiz I, Seyidova A. Clinical outcomes and prognostic factors associated with the response to erlotinib in non-small-cell lung cancer patients with unknown EGFR mutational status. Asian Pac J Cancer Prev. 2013;14(5):3255-3261.

2. Tan PS, Bilger M, de Lima Lopes G, Acharyya S, Haaland B. Metaanalysis of first-line therapies with maintenance regimens for advanced non-small-cell lung cancer (NSCLC) in molecularly and clinically selected populations. Cancer Med. 2017;6(8):1847-1860.

3. Lee JG, Wu R. Combination erlotinib-cisplatin and Atg3-mediated autophagy in erlotinib resistant lung cancer. PLoS One. 2012;7(10): e48532.

4. Kirane A, Toombs JE, Ostapoff K, et al. Apricoxib, a novel inhibitor of COX-2, markedly improves standard therapy response in molecularly defined models of pancreatic cancer. Clin Cancer Res. 2012;18(18): 5031-5042.

5. Makrilia N, Lappa T, Xyla V, Nikolaidis I, Syrigos K. The role of angiogenesis in solid tumours: an overview. Eur J Intern Med. 2009;20(7): 663-671.

6. Sutiman N, Zhang Z, Tan EH, et al. Phase I study of oral vinorelbine in combination with erlotinib in advanced non-small cell lung cancer (NSCLC) using two different schedules. PLoS One. 2016; 11(5):e0154316.
7. Kumar R, Lu SK, Minchom A, et al. A phase $1 \mathrm{~b}$ trial of the combination of an all-oral regimen of capecitabine and erlotinib in advanced non-small cell lung cancer in Caucasian patients. Cancer Chemother Pharmacol. 2016;77(2):375-383.

8. Bertrand N, Wu J, Xu X, Kamaly N, Farokhzad OC. Cancer nanotechnology: the impact of passive and active targeting in the era of modern cancer biology. Adv Drug Deliv Rev. 2014;66:2-25.

9. Loira-Pastoriza C, Todoroff J, Vanbever R. Delivery strategies for sustained drug release in the lungs. Adv Drug Deliv Rev. 2014;75:81-91.

10. Jiang T, Mo R, Bellotti A, et al. Gel-liposome-mediated co-delivery of anticancer membrane-associated proteins and small-molecule drugs for enhanced therapeutic efficacy. Adv Funct Mater. 2014;24: 2295-2304.

11. Liu K, Dai L, Li C, et al. Self-assembled targeted nanoparticles based on transferrin-modified eight-arm-polyethylene glycol-dihydroartemisinin conjugate. Sci Rep. 2016;6:29461.

12. Merino S, Martín C, Kostarelos K, Prato M, Vázquez E. Nanocomposite hydrogels: 3D polymer-nanoparticle synergies for on-demand drug delivery. ACS Nano. 2015;9(5):4686-4697.

13. Li J, Di Y, Jin C, et al. Gemcitabine-loaded albumin nanospheres (GEMANPs) inhibit PANC-1 cells in vitro and in vivo. Nanoscale Res Lett. 2013;8(1): 176 .

14. Pedrosa SS, Pereira P, Correia A, Gama FM. Targetability of hyaluronic acid nanogelto cancer cells: in vitro and in vivo studies. Eur J Pharm Sci. 2017;104:102-113.

15. Guo CY, Yan C, Luo L, et al. Enhanced expression of PKM2 associates with the biological properties of cancer stem cells from A549 human lung cancer cells. Oncol Rep. 2017;37(4):2161-2166.

16. Langer K, Balthasar S, Vogel V, Dinauer N, von Briesen H, Schubert D. Optimization of the preparation process for human serum albumin (HSA) nanoparticles. Int J Pharm. 2003;257(1-2):169-180.

17. Kratz F. Albumin as a drug carrier: design of prodrugs, drug conjugates and nanoparticles. J Control Release. 2008;132(3):171-183.

18. Kouchakzadeh H, Shojaosadati SA, Tahmasebi F, Shokri F. Optimization of an anti-HER2 monoclonal antibody targeted delivery system using PEGylated human serum albumin nanoparticles. Int $J$ Pharm. 2013;447(1-2):62-69.

19. Yu Z, Yu M, Zhang Z, Hong G, Xiong Q. Bovine serum albumin nanoparticles as controlled release carrier for local drug delivery to the inner ear. Nanoscale Res Lett. 2014;9(1):1-7. 
20. Yao Q, Zheng Y, Cheng W, et al. Difunctional fluorescent HSA modified $\mathrm{CoFe} 2 \mathrm{O} 4$ magnetic nanoparticles for cell imaging. J Mater Chem B. 2016;4(38):6344-6349.

21. Look J, Wilhelm N, von Briesen H, et al. Ligand-modified human serum albumin nanoparticles for enhanced gene delivery. Mol Pharm. 2105;12(9):3202-3213.

22. Liu F, Mu J, Xing B. Recent advances on the development of pharmacotherapeutic agents on the basis of human serum albumin. Curr Pharm Des. 2015;21(14):1866-1888.

23. Dey A, Koli U, Dandekar P, et al. Investigating behaviour of polymers in nanoparticles of Chitosan oligosaccharides coated with hyaluronic acid. Polymer. 2016;93:44-52.

24. Gotte M, Yip GW. Heparanase, hyaluronan, and CD44 in cancers: a breast carcinoma perspective. Cancer Res. 2006;66(21):10233-10237.

25. Song JM, Molla K, Anandharaj A, et al. Triptolide suppresses the in vitro and in vivo growth of lung cancer cells by targeting hyaluronan-CD44/ RHAMM signaling. Oncotarget. 2017;8(16):26927-26940.
26. Nurwidya F, Takahashi F, Kato M, et al. CD44 silencing decreases the expression of stem cell-related factors induced by transforming growth factor $\beta 1$ and tumor necrosis factor $\alpha$ in lung cancer: preliminary findings. Bosn J Basic Med Sci. 2017;17(3):228-234.

27. Piao L, Li H, Teng L, et al. Human serum albumin-coated lipid nanoparticles for delivery of siRNA to breast cancer. Nanomedicine. 2013; $9(1): 122-129$

28. Yang XY, Li YX, Li M, Zhang L, Feng LX, Zhang N. Hyaluronic acidcoated nanostructured lipid carriers for targeting paclitaxel to cancer. Cancer Lett. 2013;334(2):338-345.

29. Moore JW, Flanner HH. Mathematical comparison of dissolution profiles. Pharm Technol. 1996;20:64-74.

\section{Publish your work in this journal}

Drug Design, Development and Therapy is an international, peerreviewed open-access journal that spans the spectrum of drug design and development through to clinical applications. Clinical outcomes, patient safety, and programs for the development and effective, safe, and sustained use of medicines are the features of the journal, which has also been accepted for indexing on PubMed Central. The manuscript management system is completely online and includes a very quick and fair peer-review system, which is all easy to use. Visit http://www.dovepress.com/testimonials.php to read real quotes from published authors.

Submit your manuscript here: http://www.dovepress.com/drug-design-development-and-therapy-journal 